For Publisher's use

\title{
DESIGN AND CONSTRUCTION OF A TPC USING GEM FOILS FOR GAS AMPLIFICATION
}

\author{
PETER WIENEMANN \\ Deutsches Elektronen-Synchrotron \\ Notkestr. 85, 22607 Hamburg, Germany \\ E-mail: peter.wienemann@desy.de
}

\begin{abstract}
The challenging physics program at the International Linear Collider (ILC) poses stringent requirements on the performance of its tracking system. A large volume time projection chamber (TPC) is considered a good candidate for such a tracker. Whereas conventional TPCs used a wired based gas amplification system, a future TPC is likely to make use of micro pattern gas detectors as e. g. gas electron multipliers (GEMs) for gas amplification. This talk gives an overview over recent achievements from the R\&D activities to build a TPC with a GEM based gas amplification system. This includes charge transfer studies through multiple GEM structures, field cage design and spatial resolution measurements in high magnetic fields.
\end{abstract}

\section{Introduction}

The particle physics community recently agreed on building an $\mathrm{e}^{+} \mathrm{e}^{-}$linear collider with superconducting accelerating structures in a joint global effort. The ambitious physics program at this International Linear Collider (ILC) poses stringent requirements on the precision of its tracker as part of a precise overall detector. The measurement of the Higgs properties for example requires excellent momentum resolution for mass reconstruction and good particle identification for branching ratio measurements. A large volume time projection chamber (TPC) as for example proposed for the TESLA detector ${ }^{1}$ is considered a promising candidate as central tracking device for a detector at ILC. Contrary to conventional TPCs with a multiwire proportional chamber (MWPC) technique for gas amplification, future TPCs are likely to make use of micro pattern gas detectors (MPGDs). The best known representatives of such MPGDs are gas electron multipliers (GEMs) ${ }^{2}$ and micromegas ${ }^{3}$. MPGDs have amplification structures of order $100 \mu \mathrm{m}$ giving rise to only tiny $\vec{E} \times \vec{B}$ effects, provide a fast and narrow electron signal and have intrinsic ion feedback suppression - all features making them good candidates as gas ampli- fication system of a TPC.

In the following, some of the $\mathrm{R} \& \mathrm{D}$ activities are described which are carried out to show that TPCs equipped with GEMs meet the challenging performance requirements and to prove that they can be operated reliably. The results presented here were obtained from of a joint $R \& D$ program of various institutes from around the world. This linear collider TPC group includes groups from Aachen, Berkeley, Carleton, Cracow, DESY, Hamburg, Karlsruhe, MIT, Montreal, MPI Munich, NIKHEF, Novosibirsk, Orsay, St. Petersburg, Rostock, Saclay and Victoria.

\section{Charge Transfer through Triple GEM Structures}

A crucial item is to understand and to optimize the charge transfer through GEM structures. The goal is to choose voltage settings which allow maximal electron transparency to ensure good spatial resolution and $d E / d x$ accuracy, and in addition minimal ion transparency to keep drift field distortions due to backdrifting ions small. Charge transfer parameters like electron/ion collection/extraction efficiency and gain were measured for various voltage settings from the currents measured on the various electrodes 
of a small test chamber being irradiated by an ${ }^{55} \mathrm{Fe}$ source. In order to take effects of a magnetic field into account the measurements were performed in a superconducting magnet providing fields up to $5 \mathrm{~T}$. The parametrizations obtained from these measurements allow the minimization of the ion backdrift (ratio of the cathode and the anode current) in a convenient way by scanning the available parameter space using a computer program. Figure 1 shows the measured ion back-

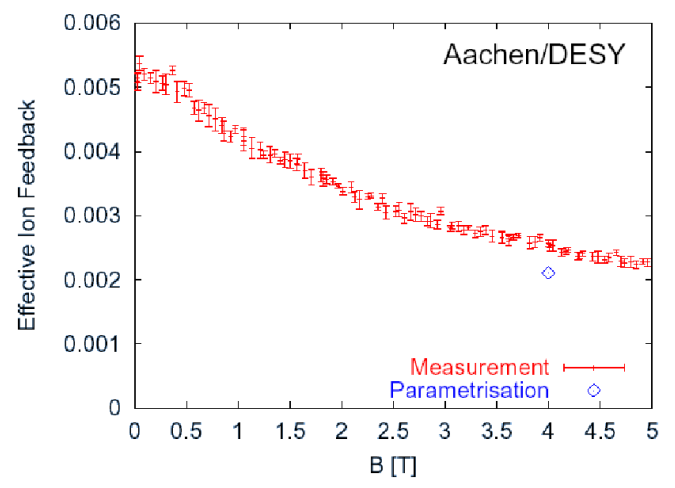

Figure 1. The measured ion backdrift versus magnetic field. In addition the predicted ion backdrift value from the parametrization is indicated.

drift versus the magnetic field. The ion backdrift decreases by a factor of two from 0 to $4 \mathrm{~T}$. A value of $0.25 \%$ is measured and corresponds well with the prediction from the parametrization. Optimally

$$
\text { ion backdrift } \times \text { gain }<1 \text {, }
$$

i. e. the number of backdrifting ions from the amplification system is less than the number of unavoidable primary ions produced in the drift region. For the achieved ion backdrift this corresponds to running with a gain of less than 400 which is probably not feasible even with new readout electronics developments. Therefore additional techniques have been attempted to further suppress ion backdrift. First tests were performed with the first GEM replaced by a micro hole strip plate $(\mathrm{MHSP})^{4}$. It was demonstrated in a first proof of principle (see Fig. 2) that by applying a negative voltage of $150 \mathrm{~V}$ to the MHSP strips the ion backdrift can be reduced by a factor of four. In order to exploit the full potential of these devices, an optimization similar to that for GEMs remains to be done.

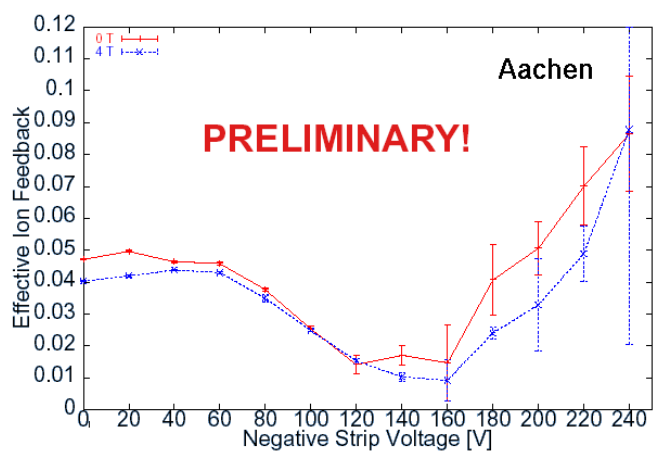

Figure 2. The ion backdrift versus the negative voltage applied to the MHSP strips.

\section{$3 \quad$ Field Cage Design}

The field cage has to meet challenging requirements. First of all, it has to provide a homogeneous electric field in order to avoid track distortions. Second, a stable mechanical support structure is needed to ensure a precise mutual alignment of the various TPC components. Third, the material budget in terms of radiation lengths has to be kept small in order to minimize a degradation of the calorimeter performance. The resistor chain needed to gradually degrade the potential from the cathode to the anode should dissipate as little heat as possible into the chamber gas because local temperature fluctuations change the drift velocity and various other gas parameters. Finally the field cage of a large-scale TPC has to stand cathode voltages of order 50 to $100 \mathrm{kV}$.

The electric field homogeneity is mainly determined by the chosen strip layout. In order to find an optimal setup, simulations have been performed with the MAXWELL 
finite element package ${ }^{5}$. Figure 3 shows the simulated relative $E$ field homogeneity for field cage designs with and without mirror strips on the outer side of the field cage. The double-sided strip layout provides inhomogeneities $\Delta E / E<10^{-4}$ which is an order of magnitude smaller than what is obtained without mirror strips on the outside.
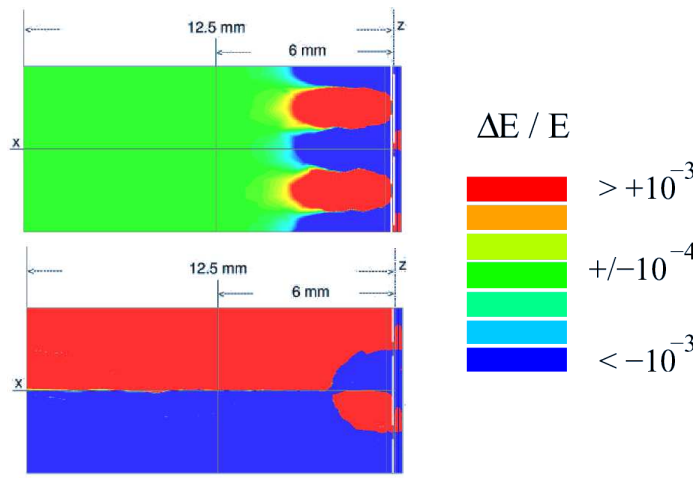

Figure 3. The simulated electric field homogeneities for field cages with (top) and without (bottom) mirror strips on the outer side of the field cage.

Several older TPC prototypes in use by the LC TPC group have only strips on the inner field cage side. The findings from the simulation study lead to the construction of a TPC prototype with a double-sided strip field cage. Its mechanical support structure is composed of honeycomb and glass-fiber reinforced plastic. Electrical insulation is provided by four layers of Kapton. In total the field cage represents only about $1 \%$ of a radiation length. It has proven to stand at least $30 \mathrm{kV}$. To reduce the heat emission of the resistor chain into the chamber gas, the resistor chain has been placed outside the gas volume. It is covered by a ceramics plate conducting the produced heat to the outside and, at the same time, providing good electric insulation. Following the careful design and test phase, the prototype performance is currently checked in first measurements with cosmic muons and a ${ }^{90} \mathrm{Sr}$ source.

\section{Transverse Resolution in High Magnetic Fields}

To achieve a high momentum resolution, a good spatial resolution is essential. The best parameter to compare the performance of different prototypes and to extrapolate to largescale devices is the single point resolution. The narrow MPGD electron signals pose a challenge to accurately reconstruct the track position with a reasonable number of channels. As opposed to micromegas, GEMs offer a nice solution. The large diffusion between the individual GEMs of a multiple GEM structure spreads the charge over a wider area without sacrificing the track resolution since the defocussing takes place during and after the gain stage. The challenge is to find GEM settings and a gas which provide a good compromise between low diffusion in the drift region and enough defocussing between the GEMs without severely degrading the two-track resolution. Several transverse resolution measurements were performed in magnetic fields up to $5 \mathrm{~T}$ both for $\mathrm{Ar}-\mathrm{CH}_{4}$ (95-5) and $\mathrm{Ar}-\mathrm{CH}_{4}-\mathrm{CO}_{2}$ (93-5-2). Figure 4

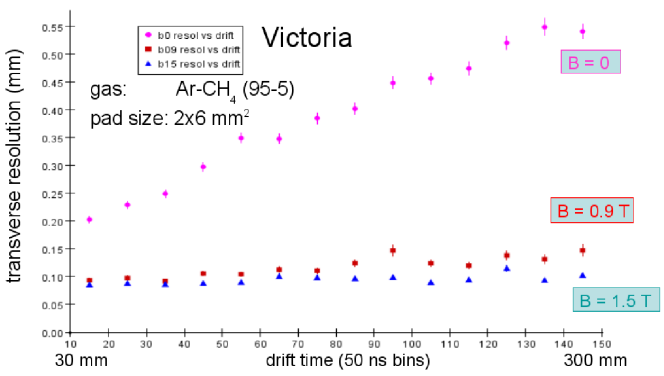

Figure 4 . The transverse resolution versus drift distance in $\mathrm{Ar}_{-} \mathrm{CH}_{4}(95-5)$.

shows the results as a function of the drift distance for Ar- $\mathrm{CH}_{4}(95-5)$ with $2 \times 6 \mathrm{~mm}^{2}$ pads. The spatial resolution becomes better with increasing magnetic fields since the $B$ field suppresses diffusion leading to narrower charge distributions arriving at the gas amplification system. Already at $1.5 \mathrm{~T}$ values 
below the $100 \mu \mathrm{m}$ level are achieved fulfilling the performance goals mentioned in the TESLA technical design report ${ }^{1}$.

\section{Conclusion}

The linear collider TPC R\&D activities of the last few years have led to valuable new insights into the properties and the potential of GEMs as gas amplification devices in TPCs. Good understanding of the charge transfer processes in multiple GEM structures has been gained. First important experiences have been made with building field cages resulting in an increasing field cage quality in the course of time. Furthermore measurements performed with such small prototypes revealed that single point resolutions of the order of $100 \mu \mathrm{m}$ are feasible for drift distance below $30 \mathrm{~cm}$ with $2 \times 6 \mathrm{~mm}^{2}$ pads. In summary, promising results have been achieved with small prototypes. Further studies are needed to show that they hold also for largescale devices.

\section{Acknowledgments}

The author would like to thank the members of the linear collider TPC group for providing their latest results.

\section{References}

1. T. Behnke, S. Bertolucci, R.-D. Heuer, and R. Settles, TESLA Technical Design Report, DESY, Hamburg, Germany, DESY 2001-011 and ECFA 2001209 (2001).

2. F. Sauli, Nucl. Instr. Meth. A 386531 (1997).

3. Y. Giomataris et al., Nucl. Instr. Meth. A 376, 29 (1996).

4. J. F. C. A. Veloso et al., Review of Scientific Instruments 71, 2371 (2000);

J. M. Maia et al., IEEE Trans. Nucl. Sci. 49 (2002).
5. MAXWELL, Finite Element Computation Package, Ansoft Co., Pittsburgh, PA. 